\title{
Regional Boundary Gradient Detectability in Distributed Parameter Systems
}

\author{
Raheam A. Al-Saphory ${ }^{1}$ and Mrooj Al-Bayati ${ }^{2}$ \\ ${ }^{1}$ Department of Mathematics, College of Education for Pure Sciences, Tikrit University, Iraq \\ ${ }^{2}$ Department of Mathematics, College of Education for Mathematics, Tikrit University, Iraq \\ ${ }^{1}$ Email: saphory@hotmail.com, ${ }^{2}$ mroojal.bayati@hotmail.com
}

\begin{abstract}
The aim of this paper is study and explore the notion of the regional boundary gradient detectability in connection with the choice of strategic gradient sensors on sub-region of the considered system domain boundary. More precisely, the principal reason behind introducing this notion is that the possibility to design a dynamic system (may be called regional boundary gradient observer) which enable to estimate the unknown system state gradient. Then for linear infinite dimensional systems in a Hilbert space, we give various new results related with different measurements. In addition, we provided a description of the regional boundary gradient strategic sensors for completion the regional boundary gradient observability and regional boundary gradient detectability. Finally, we present and illustrate some applications of sensors structures which relate by regional boundary gradient detectability in diffusion distributed parameter systems.
\end{abstract}

Keywords: $\boldsymbol{\Gamma}_{\boldsymbol{G}}$-strategic sensors, $\boldsymbol{\Gamma}_{\boldsymbol{G}}$-observer, $\boldsymbol{\Gamma}_{\boldsymbol{G}}$-detectability, infinite dimensional systems, Hilbert space.

Subject Classification: 93A30; 93B07; 93C05; 93C20.

Supporting Agencies: Tikrit University

Language: English

Date of Submission: 2018-06-14

Date of Acceptance: 2018-06-20

Date of Publication: 2018-06-30

DOI 10.24297/jam.v14i2.7478

ISSN: 2347-1921

Volume: 14 Issue: 2

Journal: Journal of Advances in Mathematics

Website: https://cirworld.com

This work is licensed under a Creative Commons Attribution 4.0 International License. 


\section{Introduction}

Many real problems in the sensors and detectability of distributed parameter systems can be reformulated as problems of infinite dimensional systems in a domain $\Omega$ [1-3]. The analysis of distributed parameter systems consists a set of notions as observability, detectability, stability, and observer is represented by infinite dimensional systems of partial differential equations [4-8]. Recently, the concept of regional and regional boundary analysis has been introduced by El Jai, Zerrik, Al-Saphory et al., for finite time horizon [9-13] and for infinite time horizon [14-16]. Thus, this concept which is given important tool to solve many problems in real world not in total domain of the considered system state but in sub-region of the domain or of the boundary [17-19]. Later, another direction of regional analysis has been extended is the system state gradient for observability, detectability and strategic sensors [20-26].

The purpose of this paper is to study and investigate the concept of regional boundary gradient detectability by using the choice of sensors. The principle reason for considering this case is that, firstly exist systems which are detectable on some boundary sub-region but are not detectable in any neighborhood of $\Gamma \subset \partial \Omega$. Secondly, it is closer to a real situation, the treatment of water by using a bioreactor where the objective is to detect the concentration of substrate at the boundary output of the bioreactor in order the water regulation is achieved (for example see figure 1) [27].

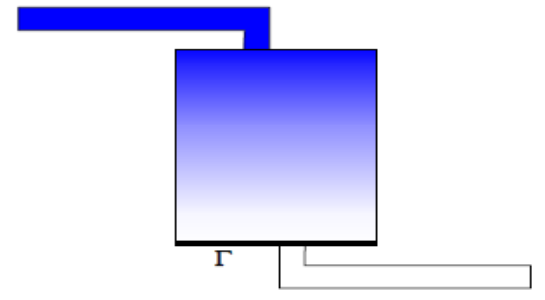

Fig. 1: Detection of substrate concentration at the boundary output of the reactor.

The outline of this paper is organized as follow.

Section 2 concerns the class of considered system, definition, characterizations in connection with sensors and preliminaries of regional boundary gradient observability and detectability. Section 3, devotes to the problem of crossing method from internal region to boundary case by using trace operator estime the gradient of state in region $\Gamma$. Section 4, gives an applications to various situations of sensors locations on the regional boundary gradient detectability in diffusion parabolic distributed systems. Finally, section tackles the relation between regional boundary detectability of state gradient and regional boundary observer.

\section{Regional Boundary Gradient Detectability}

In this section, we extend the results in $[15,21]$ to the boundary regional case by considering $\Gamma \subset \partial \Omega$. Thus, we will give some definitions which will be used to explain the notion of regional boundary gradient observability and detectability in $\left(H^{1 / 2}(\Gamma)\right)^{n}$ (state space).

\subsection{Considered system and preliminary}

Let $\Omega$ be an open bounded subset of $R^{n}$ with smooth boundary $\partial \Omega$ and $\Gamma$ be a sub-boundary of $\partial \Omega$. We considered the parabolic system is described by the following state space equations

$$
\left\{\begin{array}{lc}
\frac{\partial z}{\partial t}(\mu, t)=A z(\mu, t)+B u(t) & \Omega \times] 0, T[ \\
z(\mu, 0)=z_{0}(\mu) & \bar{\Omega} \\
\frac{\partial z}{\partial v}(\eta, t)=0 & \partial \Omega \times] 0, T[
\end{array}\right.
$$


where $\mu \in \Omega, \eta \in \partial \Omega, t \in[0, T]$ and $(\mu, t) \in \Omega \times] 0, T[,(\eta, t) \in \partial \Omega \times] 0, T[,(\mu, 0) \in \bar{\Omega}$,

Augmented with the output function

$$
y(., t)=C z(., t)
$$

- The Hilbert spaces $Z, U$ and $\mathcal{O}$ are separable where $Z=H^{1}(\bar{\Omega})$ is the state space, $\bar{\Omega}$ is the closure of $\Omega, U=$ $L^{2}\left(0, T, R^{p}\right)$ is the control space and $\mathcal{O}=L^{2}\left(0, T, R^{q}\right)$ is the observation space, where $p$ and $q$ are the numbers of actuators and sensors.

- $A=\sum_{i, j=1}^{n} \frac{\partial}{\partial z_{j}}\left(a_{i j} \frac{\partial}{\partial z_{j}}\right)$ with $a_{i j} \in D(\bar{A})$ (domain of $\bar{A}$ ) is a second order linear differential operator, which generates a strongly continuous semi-group $\left(S_{A}(t)\right)_{t \geq 0}$ on the state space $Z$ and is self-adjoint with compact resolvent.

- The operators $B \in L\left(R^{p}, Z\right)$ and $C \in L\left(Z, R^{q}\right)$ are depend on the structure of actuators and sensors as in [8] see (figure 2) which is a mathematical model is more general spatial case in (figure 1 ).

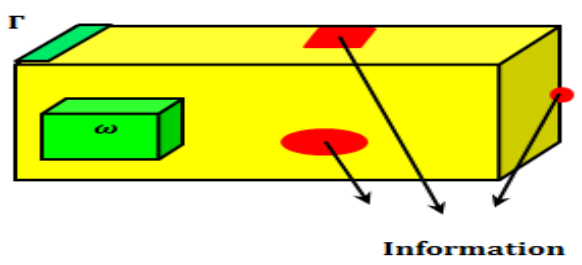

Fig. 2: The domain $\Omega$, the boundary regions $\Gamma$, and sensors locations.

- Under the given assumption above, the system (1) has a unique solution given by the following form [1-2].

$$
z(\mu, t)=S_{A}(t) z_{0}(\mu)+\int_{0}^{t} S_{A}(t-s) B u(s) d s
$$

- The problem is how to detect the current state in a given sub-boundary $\Gamma$, and to give a sufficient condition for the existence of a regional boundary gradient detectability.

- The measurements can be obtained by the use of zone or pointwise sensors, which may be located in $\Omega$ or $\partial \Omega$ [3].

- We first recall a sensors are defined by any couple $\left(D_{i}, f_{i}\right)_{1 \leq i \leq q}$ where $D_{i}$ be a non-empty closed subsets of $\bar{\Omega}$, which is spatial supports of sensors and $f_{i} \in L^{2}\left(D_{i}\right)$ represent the distributions of the sensing measurements on $D_{i}$. Then, according to the choice of the parameters $D_{i}$ and $f_{i}$, we have different types of sensors:

- It may be zone, if $D_{i} \subset \bar{\Omega}$ and $f_{i} \in L^{2}\left(D_{i}\right)$. In this case, the operator $C$ is bounded and the output function (2) may be given by the form

$$
y(t)=\int_{D_{i}} z(\mu, t) f_{i}(\mu) d \mu
$$

- It may be pointwise, if $D_{i}=\left\{b_{i}\right\}$ with $b_{i} \in \bar{\Omega}$ and $f=\delta\left(.-b_{i}\right)$, where $\delta$ is the Dirac mass concentrated in $b$. In this case, the operator $C$ is un bounded and the output function (2) may be given by the form

$$
y(t)=\int_{\Omega} z(\mu, t) \delta_{b_{i}}\left(\mu-b_{i}\right) d \mu
$$

- It may be boundary zone, if $\Gamma_{i} \subset \partial \Omega$ and $f_{i} \in L^{2}\left(\Gamma_{i}\right)$, the output function (2) may be given by the form 


$$
y(t)=\int_{\Gamma_{i}} z(\eta, t) f_{i}(\eta) d \eta
$$

- The initial state $z_{0}$ and its gradient $\nabla z_{0}$ are supposed to be unknown, the problem concerns the reconstruction of the initial gradient $\nabla z_{0}$ on the sub-region $\Gamma$ of the system domain $\partial \Omega$.

- Now, we consider the operator $K$ given by the form

$$
\begin{aligned}
& K: Z \rightarrow \mathcal{O} \\
& z \rightarrow C S_{A}(.) z
\end{aligned}
$$

where $K$ is bounded linear operator as in [7-8]. Thus, the adjoint operator $K^{*}$ of $K$ is defined by

$$
\begin{aligned}
& K^{*}: \mathcal{O} \rightarrow Z, \text { and represntedby the form } \\
& K^{*} y^{*}=\int_{0}^{t} S_{A}^{*}(s) C^{*} y^{*}(s) d s
\end{aligned}
$$

- The operator $\nabla$ denotes the gradient is given by

$$
\left\{\begin{array}{l}
\nabla: H^{1}(\Omega) \rightarrow\left(H^{1}(\Omega)\right)^{n} \\
z \rightarrow \nabla_{z}=\left(\frac{\partial z}{\partial \mu_{1}}, \ldots, \frac{\partial z}{\partial \mu_{n}}\right) .
\end{array}\right.
$$

with the adjoint of $\nabla$ denotes by $\nabla^{*}$ is given by

$$
\left\{\begin{array}{l}
\nabla^{*}:\left(H^{1}(\Omega)\right)^{n} \rightarrow H^{1}(\Omega) \\
z \rightarrow \nabla_{z}^{*}=v
\end{array}\right.
$$

where $v$ is a solution of the Dirichlet problem

$$
\begin{cases}\Delta_{v}=-\operatorname{div}(z) & \text { in } \Omega \\ v=0 & \text { in } \partial \Omega\end{cases}
$$

- The trace operator of order zero is described by [28]

$$
\gamma_{0}: H^{1}(\Omega) \rightarrow H^{1 / 2}(\partial \Omega)
$$

which is linear, subjective and continuous [2]. Thus, the extension of the trace operator of order zero which is denoted by $\gamma$ defined as

$$
\gamma:\left(H^{1}(\Omega)\right)^{n} \rightarrow\left(H^{1 / 2}(\partial \Omega)\right)^{n}
$$

and the adjoints are respectively given by $\gamma_{0}{ }^{*}, \gamma^{*}$.

- For a sub-boundary $\Gamma$ of $\partial \Omega$ and let $\tilde{\chi}_{\Gamma}$ be the function defined by

$$
\left\{\begin{array}{l}
\tilde{\chi}_{\Gamma}: H^{1 / 2}(\partial \Omega) \rightarrow H^{1 / 2}(\Gamma) \\
z \rightarrow \chi_{\Gamma} z=\left.z\right|_{\Gamma}
\end{array}\right.
$$

With $\left.z\right|_{\Gamma}$ is the restriction of the state $z$ to $\Gamma$, and

$$
\chi_{\Gamma}:\left(H^{1 / 2}(\partial \Omega)\right)^{n} \rightarrow\left(H^{1 / 2}(\Gamma)\right)^{n}
$$

where the adjoints are respectively given by $\tilde{\chi}_{\Gamma}^{*}, \chi_{\Gamma}^{*}$ 
- Finally, we introduced the operator $H_{\Gamma_{G}}=\chi_{\Gamma} \gamma \nabla K^{*}$ from $\mathcal{O}$ into $\left(H^{1 / 2}(\Gamma)\right)^{n}$ and the adjoint of this operator given by $H_{\Gamma_{G}}^{*}=K \nabla^{*} \gamma^{*} \chi_{\Gamma}^{*}$.

\subsection{Definitions and characterizations}

In this sub-section, we introduce some definitions and descriptions of regional boundary gradient observability, detectability and strategic sensors, which is derived of [20-26]. Consider the autonomous system of (1) define by

$$
\left\{\begin{array}{lc}
\frac{\partial z}{\partial t}(\mu, t)=A z(\mu, t) & \Omega \times] 0, T[ \\
z(\mu, 0)=z_{0}(\mu) & \bar{\Omega} \\
\frac{\partial z}{\partial v}(\eta, t)=0 & \partial \Omega \times] 0, T[
\end{array}\right.
$$

The solution of (7) is given by the following form

$$
z(\mu, t)=S_{A}(t)_{0}(\mu) \quad \text { for all } t \in[0, T]
$$

Definition 2.1: (a) The system (7) augmented with the output function (2) (or the systems (7)-(2)) are said to be an exactly regionally boundary gradient observable on $\Gamma$, if

$$
\operatorname{Im} H_{\Gamma_{G}}=\left(H^{1 / 2}(\Gamma)\right)^{n}
$$

(b) The systems (7)-(2) are said to be an approximately regionally boundary gradient observable on $\Gamma$, if

$$
\left.\overline{\operatorname{Im} H_{\Gamma_{G}}}=\left(H^{1 / 2}(\Gamma)\right)^{n}\right)
$$

Now, we give a notion of the regional boundary gradient strategic sensors.

Definition 2.2: A sensor $(D, f)$ is said to be regionally boundary gradient strategic on $\Gamma$ (or $\Gamma_{G}$-strategic), if the observed systems are an approximately $\Gamma_{G}$-observable.

Definition 2.3: The semi-group $\left(S_{A}(t)\right)_{t \geq 0}$ is said to be regionally boundary gradient stable (or $\Gamma_{G}$-stable) on the space $\left(H^{1 / 2}(\Gamma)\right)^{n}$, if for some positive constants $M_{\Gamma_{G}}$ and $\omega_{\Gamma_{G}}$, then

$$
\left\|\chi_{\Gamma} \gamma \nabla S_{A}(.)\right\|_{L\left(\left(H^{1 / 2}(\Gamma)\right)^{n}, H^{1}(\bar{\Omega})\right)} \leq M_{\Gamma_{G}} e^{-\omega_{\Gamma_{G}} t}, t \geq 0
$$

Remark 2.4: If the semi-group $\left(S_{A}(t)\right)_{t \geq 0}$ is $\Gamma_{G}$-stable on $\left(H^{1 / 2}(\Gamma)\right)^{n}$, then for all $z_{0}(.) \in H^{1}(\bar{\Omega})$, the solution of the system (7) satisfies

$$
\lim _{t \rightarrow \infty}\left\|\chi_{\Gamma} \gamma \nabla z(., t)\right\|_{\left(H^{1 / 2}(\Gamma)\right)^{n}}=\lim _{t \rightarrow \infty}\left\|\chi_{\Gamma} \gamma \nabla S_{A}(t) z_{0}\right\|_{\left(H^{1 / 2}(\Gamma)\right)^{n}}=0
$$

Definition 2.5: The system (7) is $\Gamma_{G}$-stable, if the operator $A$ generates a semi-group which is $\Gamma_{G}$-stable on $\left(H^{1 / 2}(\Gamma)\right)^{n}$.

Definition 2.6: The systems (1)-(2) are said to be regionally boundary gradient detectable on $\Gamma$ (or $\Gamma_{G}$ detectable), if there exists an operator $H_{\Gamma_{G}}: R^{q} \rightarrow\left(H^{1 / 2}(\Gamma)\right)^{n}$, such that the operator $\left(A-H_{\Gamma_{G}} C\right)$ generates a strongly continuous semi-group $\left(S_{H_{\Gamma_{G}}}(t)\right)_{t \geq 0}$, which is $\Gamma_{G}$-stable on $\left(H^{1 / 2}(\Gamma)\right)^{n}$.

Proposition 2.7: If the systems (1)-(2) are an exactly $\Gamma_{G}$-observable, then it is $\Gamma_{G}$-detectable. This results gives the following inequality: $\exists k>0$, such that

$$
\left\|\chi_{\Gamma} \gamma \nabla S_{A}(.)_{Z}\right\|_{\left(H^{1 / 2}(\Gamma)\right)^{n}} \leq k\left\|C S_{A}(.) Z\right\|_{L^{2}(0, \infty, 0)}, \text { for all } z \in\left(H^{1 / 2}(\Gamma)\right)^{n}
$$


Proof: We conclude the proof of this proposition is conclude from the results on observability considering $\chi_{\Gamma} \nabla K^{*}$. We have the following forms [2]

1- $\operatorname{Imf} \subset \operatorname{Img}$.

2- There exists $k>0$, such that

$$
\left\|f^{*} z^{*}\right\|_{E^{*}} \leq k\left\|g^{*} z^{*}\right\|_{F^{*}} \text {, for all } z^{*} \in G^{*}
$$

From the right hand said of above inequality $k\left\|g^{*} z^{*}\right\|_{F^{*}}$, there exists $M_{\Gamma_{G}}, \omega_{\Gamma_{G}}>0$ with $k<M_{\Gamma_{G^{\prime}}}$ such that

$$
k\left\|g^{*} z^{*}\right\|_{F^{*}} \leq M_{\Gamma_{G}} e^{-\omega_{\Gamma_{G}} t}\left\|_{z^{*}}\right\|_{F^{*}}
$$

where $E, F$ and $G$ be a reflexive Banach spaces and $f \in L(E, G), g \in L(F, G)$. If we apply this result, considered

$$
E=G=\left(H^{1 / 2}(\Gamma)\right)^{n}, F=\mathcal{O}, f=I d_{\left(H^{1 / 2}(\Gamma)\right)^{n}}
$$

and

$$
g=S_{A}^{*}(.) \chi_{\Gamma}^{*} \gamma^{*} \nabla^{*} C^{*}
$$

where $S_{A}\left(\right.$.) is a strongly continuous semi-group generates by $A$, which is $\Gamma_{G}$-stable, then it is $\Gamma_{G}$-detectable. Thus, the notion of $\Gamma_{G}$-detectability is a weaker property than the exact $\Gamma_{G}$-observability [14-15].

\section{$2.3 \Gamma_{G}$-Strategic sensors and $\Gamma_{G}$-detectability}

In the sub-section, we shall develop the characterization result that links an $\Gamma_{G}$-detectable and sensors structures. For that purpose, we assume that the operator $A$ has a complete set of eigenfunctions $H^{1}(\bar{\Omega})$ (which is Sobolev space of order one) [1] denoted $\varphi_{m j}$ orthonormal in $\left(H^{1 / 2}(\Gamma)\right)^{n}$ and the associated eigenvalues $\lambda_{m}$ are of multiplicity $r_{m}$ and suppose that the system (1) has $J$ unstable modes.

Thus, the sufficient condition of an $\Gamma_{G}$-detectability is given by the following theorem.

Theorem 2.8: Suppose that there are $q$ zone sensors $\left(D_{i}, f_{i}\right)_{1 \leq i \leq q}$ and the spectrum of $A$ contains $J$ eigenvalues with non-negative real parts. The systems (1)-(2) are $\Gamma_{G}$-detectable if and only if

1- $q \geq m$,

2- rank $G_{i}=m_{i}$, for all $i, i=1, \ldots, J$ with

$$
G=(G)_{i j}= \begin{cases}<\psi_{j}(.), f_{i}(.)>_{L^{2}\left(D_{i}\right)} & \text { zone sensors } \\ \psi_{j}\left(b_{i}\right) & \text { pointwise sensors } \\ <\frac{\partial \psi_{j}}{\partial v}, f_{i}(.)>_{L^{2}\left(\Gamma_{i}\right)} & \text { boundary zone sensors }\end{cases}
$$

where $\sup m_{i}=m<\infty$ and $\mathrm{j}=1, \ldots, m_{i}$.

Proof: For brevity, the proof is limited to the case of zone sensors. Under the assumptions of section 2.1, the system (1) may be decomposed by the projections $P$ and $I-P$ on two parts, unstable and stable [7]. The state vector may be given by $z(\mu, t)=\left[z_{1}(\mu, t) z_{2}(\mu, t)\right]^{t r}$, where $z_{1}(\mu, t)$ is the state component of the unstable part of the system (1) that may be written in the form 


$$
\left\{\begin{array}{lc}
\frac{\partial z_{1}}{\partial t}(\mu, t)=A_{1} z_{1}(\mu, t)+P B u(t) & \Omega \times] 0, T[ \\
z_{1}(\mu, 0)=z_{1_{0}}(\mu) & \bar{\Omega} \\
\frac{\partial z_{1}}{\partial v}(\eta, t)=0 & \partial \Omega \times] 0, T[
\end{array}\right.
$$

and $z_{2}(\mu, t)$ is the component state of the stable part of the system (1) given by

$$
\left\{\begin{array}{lc}
\frac{\partial z_{2}}{\partial t}(\mu, t)=A_{2} z_{2}(\mu, t)+(I-P) B u(t) & \Omega \times] 0, T[ \\
z_{2}(\mu, 0)=z_{2_{0}}(\mu) & \bar{\Omega} \\
\frac{\partial z_{2}}{\partial v}(\eta, t)=0 & \partial \Omega \times] 0, T[
\end{array}\right.
$$

The operator $A_{1}$ is represented by a matrix of order $\left(\sum_{i=1}^{J} m_{i}, \sum_{i=1}^{J} m_{i}\right)$ defined by $A_{1}=$ $\operatorname{diag}\left[\lambda_{1}, \ldots, \lambda_{1}, \lambda_{2}, \ldots, \lambda_{2}, \ldots, \lambda_{J}, \ldots, \lambda_{J}\right]$ and $P B=\left[G_{1}^{t r}, G_{2}^{t r}, \ldots, G_{J}^{t r}\right]$. By using the condition (2) of this theorem, we conclude that the suite $\left(D_{i}, f_{i}\right)_{1 \leq i \leq q}$ of sensors are $\Gamma_{G}$-strategic for the unstable part of the system (1), the subsystem (12) is an approximately $\Gamma_{G}$-observable [23]. Since this system it is of finite dimensional, then it is an exactly $\Gamma_{G}$-observable [22]. Therefore it is $\Gamma_{G}$-detectable [21], and hence there exists an operator $H_{\Gamma_{G}}^{1}$ such that $\left(A_{1}-H_{\Gamma_{G}}^{1} C\right)$ which satisfies the following $[15,23]$ :

$$
\exists M_{\Gamma_{G}}^{1}, \omega_{\Gamma_{G}}^{1}>0 \text {, such that }\left\|e^{\left(A_{1}-H_{\Gamma_{G}}^{1} C\right) t}\right\|_{\left(H^{1 / 2}(\Gamma)\right)^{n}} \leq M_{\Gamma_{G}}^{1} e^{-\omega_{\Gamma_{G}}^{1} t}
$$

and we have

$$
\left\|z_{1}(., t)\right\|_{\left(H^{1 / 2}(\Gamma)\right)^{n}} \leq M_{\Gamma_{G}}^{1} e^{-\omega_{\Gamma_{G}}^{1} t}\left\|P z_{0}(.)\right\|_{\left(H^{1 / 2}(\Gamma)\right)^{n}} .
$$

Since the semi-group generated by the operator $A_{2}$ is $\Gamma_{G}$-stable on $\left(H^{1 / 2}(\Gamma)\right)^{n}$, then there exist $M_{\Gamma_{G}}^{2}, \omega_{\Gamma_{G}}^{2}>0$, such that

$$
\left\|z_{2}(., t)\right\|_{\left(H^{1 / 2}(\Gamma)\right)^{n}} \leq M_{\Gamma_{G}}^{2} e^{-\omega_{\Gamma G}^{2} t}\left\|(I-P) z_{0}(.)\right\|_{\left(H^{1 / 2}(\Gamma)\right)^{n}}+\int_{0}^{t} M_{\Gamma_{G}}^{2} e^{-\omega_{\Gamma_{E G}}^{2} t}\left\|(I-P) z_{0}(.)\right\|_{\left(H^{1 / 2}(\Gamma)\right)^{n}}\|u(\tau)\| d \tau
$$

Therefore $z(\mu, t) \rightarrow 0$ when $t \rightarrow \infty$. Finally, the system (1) and (2) are $\Gamma_{G}$-detectable.

Conversely, if the systems (1)-(2) are $\Gamma_{G}$-detectable. Then there exists an operator $H_{\Gamma_{G}} \in L\left(R^{q},\left(H^{1 / 2}(\Gamma)\right)^{n}\right)$, such that $\left(A-H_{\Gamma_{G}} C\right)$ generates $\Gamma_{G}$-stable, strongly continuous semi-group $\left(S_{H_{\Gamma_{G}}}(t)\right)_{t \geq 0}$ on the space $\left(H^{1 / 2}(\Gamma)\right)^{n}$ which satisfies the following:

$$
\exists M_{\Gamma_{G}}, \omega_{\Gamma_{G}}>0 \text {, such that }\left\|\chi_{\Gamma} \gamma \nabla S_{H_{\Gamma_{G}}}(.)\right\|_{\left(H^{1 / 2}(\Gamma)\right)^{n}} \leq M_{\Gamma_{G}} e^{-\omega_{\Gamma_{G}} t} .
$$

Thus, the unstable sub-system (12) is $\Gamma_{G}$-detectable. We recall that a system is an approximately $\Gamma_{G}$-observable, i.e.

$$
\left[K \gamma^{*} \nabla^{*} \chi_{\Gamma}^{*} z^{*}(., t)=0 \Rightarrow z^{*}(., t)=0\right], \text { for } z^{*}(., t) \in\left(H^{1 / 2}(\Gamma)\right)^{n},
$$

we have

$$
K \gamma^{*} \nabla^{*} \chi_{\Gamma}^{*} Z^{*}(., t)=\left(\sum_{i=1}^{J} e^{\lambda_{j} t}<\psi_{j}(.), \gamma^{*} \chi_{\Gamma}^{*} \nabla z^{*}(., t)>_{\left(H^{1 / 2}(\Gamma)\right)^{n}}<\psi_{j}(.), f_{j}(., t)>_{\left(H^{1 / 2}(\partial \Omega)\right)^{n}}\right)_{1 \leq i \leq q}
$$

If the rank $G_{i} z_{i} \neq m_{i}$ for all $i, i=1, \ldots, J$, there exists $z^{*}(., t) \in\left(H^{1 / 2}(\Gamma)\right)^{n}$, such that $K \gamma^{*} \nabla^{*} \chi_{\Gamma}^{*} z^{*}(., t)=0$, this leads 
$\sum_{j=1}^{J}<\psi_{j}(),. z^{*}(., t)>_{\left(H^{1 / 2}(\Gamma)\right)^{n}}<\psi_{j}(),. f_{j}(., t)>_{\left(H^{1 / 2}(\Gamma)\right)^{n}}=0$.

The state vectors $z_{i}$ may be given by

$$
z_{i}(., t)=\left[\left\langle\psi_{j}(.), z^{*}(., t)>_{\left(H^{1 / 2}(\Gamma)\right)^{n}}<\psi_{j}(.), z^{*}(,, t)>_{\left(H^{1 / 2}(\partial \Omega)\right)^{n}}\right]^{t r} \neq 0\right.
$$

we obtain $G_{i} z_{i}=0$ for all $i, i=1, \ldots, J$

Consequently, the sub-system (12) is not an approximately $\Gamma_{G}$-observable [23] and therefore the suite $\left(D_{i}, f_{i}\right)_{1 \leq i \leq q}$ of sensors is not $\Gamma_{G}$-strategic. Thus, the systems (1)-(2) are not $\Gamma_{G}$-detectable [22]. Finally, we have rank $G_{i} \neq m_{i}$ for all $i, i=1, \ldots, J$.

\section{Internal and Regional Boundary Detection of State Gradient Approach}

In this section, we show that it is possible to link the internal regional gradient detectability and regional boundary gradient detectability, if we consider the following

- Let $\mathcal{R}:\left(H^{1 / 2}(\Gamma)\right)^{n} \rightarrow\left(H^{1}(\Omega)\right)^{n}$, which is continuous and linear given by [28]

$$
\chi_{\Gamma} \gamma \nabla \mathcal{R} h(\mu, t)=h(\mu, t), \quad \text { for all } h \in\left(H^{1 / 2}(\Gamma)\right)^{n}
$$

- Let $E=\bigcup_{z \in \Gamma} B(z, r)$ and $\bar{\omega}_{r}=E \cap \bar{\Omega}$, where $B(z, r)$ is the ball of radius $r$ centered in $z(\mu, t)$ and $\Gamma$ is a part of $\bar{\omega}_{r}(r>0$ is an arbitrary and sufficiently small real) see (Figure 3 ).

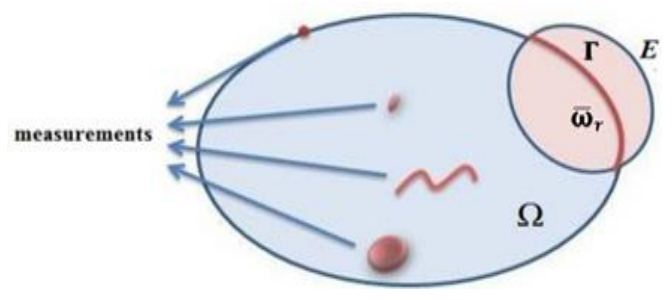

Fig. 3. The domain $\Omega$, sub-domain $\bar{\omega}_{r}$ and the region $\Gamma$.

Now, we show that there exists a link between the $\Gamma_{G}$-strategic sensor and $\bar{\omega}_{r_{G}}$-strategic sensor in the following result.

Proposition 3.1: From the above results, we deduce that.

1- A sensor is $\Gamma_{G}$-strategic if the sensor is $\bar{\omega}_{r_{G}}$-strategic.

2- A sensor is $\Gamma_{G}$-strategic if the system is exactly $\bar{\omega}_{r_{G}}$-observable.

Proof 1: Since the sensor is $\bar{\omega}_{r_{G}}$-strategic in $\bar{\omega}_{r}$, this mean that the system is approximately $\bar{\omega}_{r_{G}}$-observable in $\bar{\omega}_{r}$.

Thus, the system is approximately $\Gamma_{G}$-observable [23]. Therefore, the sensor is $\Gamma_{G}$-strategic. $\square$

Proof 2: Let $z(\mu, t) \in\left(H^{1 / 2}(\Gamma)\right)^{n}$ and $\bar{z}(\mu, t)$ be an extension to $\left(H^{1 / 2}(\partial \Omega)\right)^{n}$. By using equation (11) and trace theorem there exists $\mathcal{R} \bar{z}(\mu, t) \in\left(H^{1}(\Omega)\right)^{n}$, with bounded support such that

$$
\gamma \mathcal{R} \bar{z}(\mu, t)=\bar{z}(\mu, t) .
$$


Since the system is an exactly $\bar{\omega}_{r_{G}}$-observable, then the system is approximately $\bar{\omega}_{r_{G}}$-observable [3]. And, since a system is approximately $\bar{\omega}_{r_{G}}$-observable then a system is approximately $\Gamma_{G}$-observable $[1,21-23]$ Thus, the sensor is $\Gamma_{G}$-strategic. $\square$

Corollary 3.2: From the previous results, we have.

1- If the system is an exactly $\bar{\omega}_{r_{G}}$-observable, then the system is exactly $\Gamma_{G}$-observable, i.e., there exists an operator $\chi_{\bar{\omega}_{r}} \nabla K^{*}: \mathcal{O} \rightarrow\left(H^{1}\left(\omega_{r}\right)\right)^{n}$ given by

$$
H_{\bar{\omega}_{r}} y(., t)=\chi_{\bar{\omega}_{r}} \nabla K^{*} y(., t)=\chi_{\bar{\omega}_{r}} \mathcal{R} \bar{z}(\mu, t)
$$

Hence,

$$
\chi_{\Gamma}\left(\gamma \chi_{\bar{\omega}_{r}} \nabla K^{*} y(., t)\right)=z(\mu, t) .
$$

where $z(\mu, t) \in\left(H^{1 / 2}(\Gamma)\right)^{n}$ and $\bar{z}(\mu, t)$ be an extension to $\left(H^{1 / 2}(\partial \Omega)\right)^{n}$.

2- If the system is approximately $\bar{\omega}_{r_{G}}$-observable, then the system is approximately $\Gamma_{G}$-observable.

Definition 3.3: The system (1) is $\omega_{r_{G}}$-stable, if the solution of autonomous system associated with (1) together with (2) converges exponentially to zero when $t \rightarrow \infty$.

Definition 3.4: The systems (1)-(2) are $\omega_{r_{G}}$-detectability, if there exists an operator $H_{\omega_{r_{G}}}: \mathcal{O} \rightarrow\left(H^{1}\left(\omega_{r}\right)\right)^{n}$, such that the operator $\left(A-H_{\omega_{r_{G}}} C\right)$ generates a strongly continuous semi-group $\left(S_{H_{\omega_{r_{G}}}}(t)\right)_{t \geq 0}$, which is $\omega_{r_{G}}$-stable.

Proposition 3.5: If the systems (1)-(2) are an exactly $\bar{\omega}_{r_{G}}$-observable, then it is $\Gamma_{E G}$-detectable.

Proof: For the proof see ref. [15], with miner changment.

Now, the method of crossing from internal $\omega_{r_{G}}$-detectability into $\Gamma_{G}$-detectability will be given in the following theorem.

Theorem 3.6: If the systems (1)-(2) are $\bar{\omega}_{r_{G}}$-detectable, then it is $\Gamma_{G}$-detectable.

Proof: Let $z(\mu, t) \in\left(H^{1 / 2}(\Gamma)\right)^{n}$ and $\bar{z}(\mu, t)$ be an extension to $\left(H^{1 / 2}(\partial \Omega)\right)^{n}$ [28]. By using equation (14) and trace theorem, there exist

$$
\mathcal{R} \bar{z}(\mu, t) \in\left(H^{1}(\Omega)\right)^{n}
$$

with a bounded support, such that

$$
\gamma(\mathcal{R} \bar{z}(\mu, t))=\bar{z}(\mu, t)
$$

since the systems (1)-(2) are $\bar{\omega}_{r_{G}}$-detectable, then it is $\omega_{r_{G}}$-detectable. Thus, there exists an operator

$$
\chi_{\omega_{r}} \nabla K^{*}: \mathcal{O} \rightarrow\left(H^{1}\left(\omega_{r}\right)\right)^{n}
$$

is defined by

$$
H_{\omega_{r_{G}}} y(., t)=\chi_{\omega_{r}} \nabla K^{*} y(\mu, t)
$$


such that the operator $\left(A-H_{\omega_{r_{G}}} C\right)$ generates a strongly continuous semi-group $\left(S_{H_{\omega_{r_{G}}}}(t)\right)_{t \geq 0}$, which is $\omega_{r_{G}}{ }^{-}$ stable. For every $y \in \mathcal{O}$, then we obtain

$$
\chi_{\omega_{r}} \nabla K^{*} y(\mu, t)=\chi_{\omega_{r}} \nabla \mathcal{R} \bar{z}(\mu, t)
$$

and hence

$$
\chi_{\Gamma}\left(\gamma \nabla \chi_{\omega_{r}} K^{*} y\right)(., t)=z(\mu, t)
$$

Consequently, there exists an operator

$$
H_{\Gamma_{G}}=\chi_{\Gamma}\left(\gamma \nabla \chi_{\omega_{r}} K^{*} y\right): \mathcal{O} \rightarrow\left(H^{1 / 2}(\Gamma)\right)^{n},
$$

such that $\left(A-H_{\Gamma_{G}} C\right)$ generates a semi-group $\left(S_{H_{\Gamma_{G}}}(t)\right)_{t \geq 0}$, which is $\Gamma_{G}$-stable.

Finally, the systems (1)-(2) are $\Gamma_{G}$-detectable [15].

\section{Application to Sensors Locations}

In this section, we will explore different results related to different types of measurements and, we give the results on the locations of internal and boundary (pointwise and zone). Consider the two dimensional of diffusion system on a rectangular domain will may be described by the following form

$$
\left\{\begin{array}{lc}
\frac{\partial z}{\partial t}\left(\mu_{1}, \mu_{2}, t\right)=\frac{\partial^{2} z}{\partial \mu_{1}^{2}}\left(\mu_{1}, \mu_{2}, t\right)+\frac{\partial^{2} z}{\partial \mu_{2}^{2}}\left(\mu_{1}, \mu_{2}, t\right)+z\left(\mu_{1}, \mu_{2}, t\right) & \Omega \times] 0, T[ \\
z\left(\mu_{1}, \mu_{2}, 0\right)=z_{0}\left(\mu_{1}, \mu_{2}\right) & \bar{\Omega} \\
\frac{\partial z}{\partial v}\left(\eta_{1}, \eta_{2}, t\right)=0 & \partial \Omega \times] 0, T[
\end{array}\right.
$$

with measurements obtained by output function given as in (2), where $\Omega=] 0, a_{1}[\times] 0, a_{2}[, \Gamma=] 0, a_{1}\left[\times\left\{a_{2}\right\}\right.$ and the eigenfunctions of the dynamic system (15) for Dirichlet boundary conditions are given by

$$
\varphi_{n m}\left(\mu_{1}, \mu_{2}\right)=\left(\frac{4}{a_{1} a_{2}}\right)^{1 / 2} \cos n \pi\left(\frac{\mu_{1}}{a_{1}}\right) \cos m \pi\left(\frac{\mu_{2}}{a_{2}}\right)
$$

associated with eigenvalues

$$
\lambda_{n m}=-\left(\frac{n^{2}}{a_{1}^{2}}+\frac{m^{2}}{a_{2}^{2}}\right) \pi^{2}, n, m \geq 1
$$

If we suppose that $a_{1}^{2} / a_{2}^{2} \notin Q$, then the multiplicity of the eigenvalues $\lambda_{n m}$ is $r_{n m}=1$ for every $n, m=\{1, \ldots, J\}$, then one sensor $(D, f)$ may be sufficient for $\Gamma_{G}$-detectable [16].

\subsection{Case of Zone Sensor}

We study and discuss the following cases.

\section{- Internal Zone Sensor}

Consider the system (15)-(2) may be given by the form

$$
y(t)=\int_{D} z\left(\mu_{1}, \mu_{2}, t\right) f\left(\mu_{1}, \mu_{2}\right) d \mu_{1} d \mu_{2}
$$


where the zone sensor is the located inside the domain $\Omega$, over the support

$$
D=] \mu_{1_{0}}-l_{1}, \mu_{1_{0}}+l_{1}[\times] \mu_{2_{0}}-l_{2}, \mu_{2_{0}}+l_{2}\left[\subset \Omega f \in L^{2}(D)\right.
$$

as in (figure 4). Now, from the above result, we give the following result.

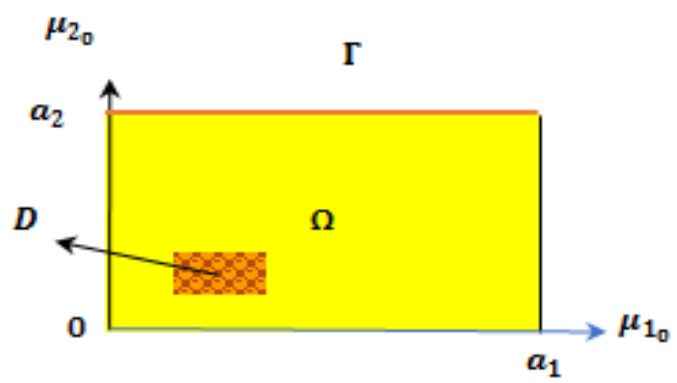

Fig. 4: Location $D$ of internal zone sensor.

Proposition 4.1: If the function $f_{i}$ is symmetric with respect to $\mu_{i}=\mu_{i_{0}}, i=1,2$, then the systems (15)-(18) are $\Gamma_{G}$-detectable, if

$$
n \mu_{1_{0}} / a_{1} \text { and } m \mu_{2_{0}} / a_{2} \notin N, \text { for all } n, m=\{1, \ldots, J\} .
$$

\section{- Boundary Zone Sensor}

Now, the measurements are given by the output

$$
y(t)=\int_{\Gamma_{0}} \frac{\partial z}{\partial v}\left(\eta_{1}, \eta_{2}, t\right) f\left(\eta_{1}, \eta_{2}\right) d \eta_{1} \eta_{2}
$$

with $\Gamma_{0} \subset \partial \Omega$ is the boundary support of the sensor and $f \in L^{2}\left(\Gamma_{0}\right)$. In the case, where the support of the sensor $(D, f)$ is one of side as in (figure 5$)$, then we have the following proposition.
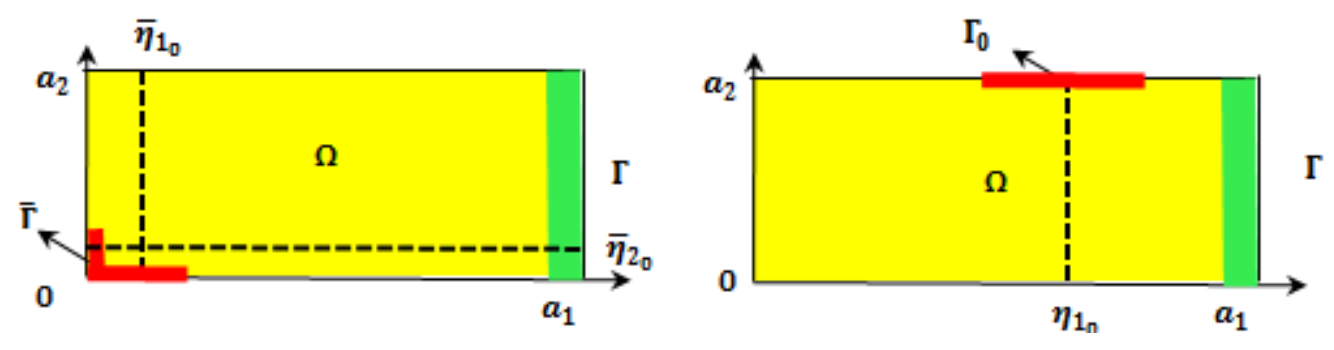

Fig. 5: Locations $\Gamma_{0}, \bar{\Gamma}$ of boundary zone sensors.

Proposition 4.2: If the function $f$ is symmetric with respect to $\eta_{1}=\eta_{1_{0}}$, then the systems (15)-(19) are $\Gamma_{G}$ detectable, if

$$
n \eta_{1_{0}} / a_{1} \notin N, \text { for all } n, n=\{1, \ldots, J\}
$$

when the support of the sensor is on two sides, i.e., $\bar{\Gamma}=\left[0, \bar{\eta}_{1_{0}}+l_{1}\right] \times\{0\} \cup\{0\} \times\left[0, \bar{\eta}_{2_{0}}+l_{2}\right]=\Gamma_{1} \cup \Gamma_{2} \subset \partial \Omega$, as in (figure 5) we obtain the following result.

Proposition 4.3: If the function $f_{i}$ is symmetric with respect to $\eta_{i}=\bar{\eta}_{i_{0}}, i=1,2$, then the systems (15)-(19) are $\Gamma_{G}$-detectable, if 
$2 n \bar{\eta}_{1_{0}} / a_{1}$ and $2 m \bar{\eta}_{2_{0}} / a_{2} \notin N$, for all $n, m=\{1, \ldots, J\}$.

\subsection{Case of Pointwise Sensor}

We investigate different pointwise cases.

\section{- Internal Pointwise Sensor}

In this case, we can give the output function by the following form

$$
y(t)=\int_{\Omega} z\left(\mu_{1}, \mu_{2}, t\right) \delta\left(\mu_{1}-b_{1}, \mu_{2}-b_{2}\right) d \mu_{1} d \mu_{2}
$$

where $b=\left(b_{1}, b_{2}\right)$ is the location of pointwise sensor in $\Omega$ as defined in (figure 6$)$. Then we obtain the following result.
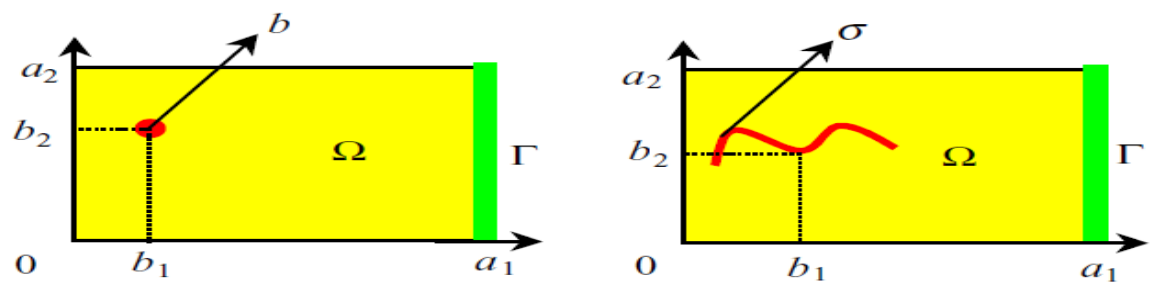

Fig. 6: Locations $b, \sigma$ of internal pointwise and filament sensors.

Proposition 4.4: We conclude that.

1-Pointwise case: If the sensor is located in $b=\left(b_{1}, b_{2}\right)$, then the systems (15)-(20) are $\Gamma_{G}$-detectable, if $n b_{1} / a_{1}$ and $m b_{2} / a_{2} \notin N$, for all $n, m=\{1, \ldots, J\}$

2- Filament case: Suppose that the observation is given by the filament sensor $\sigma=\operatorname{Im}(\gamma)$ is symmetric with respect to the line $b=\left(b_{1}, b_{2}\right)$, if

$n b_{1} / a_{1}$ and $m b_{2} / a_{2} \notin N$, for all $n, m=\{1, \ldots, J\}$.

\section{- Boundary pointwise sensor}

Suppose that the sensor $\left(b, \delta_{b}\right)$ is located on $b$, where $b=\left(b_{1}, b_{2}\right) \in \partial \Omega$ with $b=\left(0, b_{2}\right)$ as in (Figure 7).

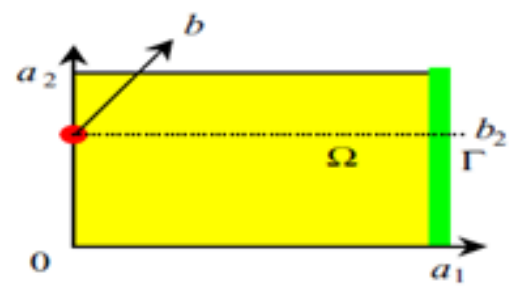

Fig. 7: Location $b$ of boundary pointwise sensor.

The output function is given by

$$
y(t)=\int_{\partial \Omega} \frac{\partial z}{\partial v}\left(0, \eta_{2}, t\right) \delta\left(0, \eta_{2}-b_{2}\right) d \eta_{1}
$$


where $b=\left(0, b_{2}\right)$. Thus, we obtain the following result.

Proposition 4.5: If the sensor support $b \in \partial \Omega$, then systems (15)-(21) are not $\Gamma_{G}$-detectable, if

$$
2 n b_{2} / a_{2} \notin N \text {, for all } n, n=\{1, \ldots, J\} .
$$

Remark 4.6: We can extend previous results in the case of rectangular domain to the case of circular domain with the following system

$$
\left\{\begin{array}{lc}
\frac{\partial z}{\partial t}(r, \theta, t)=\frac{\partial^{2} z}{\partial r^{2}}(r, \theta, t)+\frac{\partial^{2} z}{\partial \theta^{2}}(r, \theta, t)+z(r, \theta, t) & \Omega \times] 0, T[ \\
z(r, \theta, 0)=z_{0}(r, \theta) & \bar{\Omega} \\
\frac{\partial z}{\partial v}(a, \theta, t)=0 & \partial \Omega \times] 0, T[
\end{array}\right.
$$

augmented output function is defined by

$$
y_{n}(t)=\int_{D_{n}} \frac{\partial z}{\partial v}\left(r_{n}, \theta_{n}, t\right) f\left(r_{n}, \theta_{n}\right) d r_{n} d \theta_{n}
$$

where $2 \leq n \leq q, \Omega=] 0, a[, r=a>0, \theta \in[0,2 \pi]$, for zone and pointwise sensor may be internal or boundary.

\section{5. $\Gamma_{G}$-Detectability and $\Gamma_{G}$-Observer}

In this section, we show that the regional boundary gradient detectability is the possibility to defined a regional boundary gradient observer which is enable to estimate the state gradient of considered system in part $\Gamma$ of boundary $\partial \Omega$. This approach is derived from the previuos researchs as in $[7,13-16,21]$.

Definition 5.1: Suppose that there exists dynamical system with state $w(\mu, t) \in W$ given by

$$
\left\{\begin{array}{lc}
\frac{\partial w}{\partial t}(\mu, t)=F_{\Gamma_{G}} w(\mu, t)+G_{\Gamma_{G}} u(t)+H_{\Gamma_{G}} y(., t) & \Omega \times] 0, T[ \\
w(\mu, 0)=w_{0}(\mu) & \bar{\Omega} \\
\frac{\partial w}{\partial v}(\eta, t)=0 & \partial \Omega \times] 0, T[
\end{array}\right.
$$

where $F_{\Gamma_{G}}$ generates a strongly continuous semi-group, which is $\Gamma_{G}$-stable on the space $W, G_{\Gamma_{G}} \in L(U, W)$ and $H_{\Gamma_{G}} \in L(\mathcal{O}, W)$. The system (24) defines an $\Gamma_{G}$-estimator for $T_{\Gamma_{G}} z(\mu, t)=\chi_{\Gamma} \nabla T z(\mu, t)$, if the following conditions hold:

1- $\lim _{t \rightarrow \infty}\left[T_{\Gamma_{G}} z(\mu, t)-w(\mu, t)\right]=0, \quad \mu \in \Gamma$.

3- $T_{\Gamma_{G}}$ maps $D(A)$ into $D\left(F_{\Gamma_{G}}\right)$, where $z(\mu, t)$ and $w(\mu, t)$ are the solutions of systems (1)-(2) and (24).

Definition 5.2: The system (24) specifies an $\Gamma_{G}$-observer for the system (1) together with the output function (2), if the following conditions hold:

1- There exists $R_{\Gamma_{G}} \in L\left(\mathcal{O},\left(H^{1 / 2}(\Gamma)\right)^{n}\right)$ and $S_{\Gamma_{G}} \in L\left(\left(H^{1 / 2}(\Gamma)\right)^{n}\right)$, such that

$$
R_{\Gamma_{G}} C+S_{\Gamma_{G}} T_{\Gamma_{G}}=I_{\Gamma_{G}}
$$

2- $T_{\Gamma_{G}} A-F_{\Gamma_{G}} T_{\Gamma_{G}}=H_{\Gamma_{G}} C$ and $G_{\Gamma_{G}}=T_{\Gamma_{G}} B$.

3- The system (14) defines an $\Gamma_{G}$-estimator for $T_{\Gamma_{G}} z(\mu, t)$. 
Proposition 5.3: If the systems (1)-(2) are $\Gamma_{G}$-detectable, then the dynamical system (24) is $\Gamma_{G}$-observer of the systems (1)-(2), if

$$
\operatorname{Lim}_{t \rightarrow \infty}[z(\mu, t)-w(\mu, t)]=0, \quad \mu \in \Gamma
$$

Proof: Let $\varphi(\mu, t)=z(\mu, t)-w(\mu, t)$, where $w(\mu, t)$ is the solution of the system (24). Deriving the above equation and using the equations (1) and (24), we obtain

$$
\begin{aligned}
\frac{\partial \varphi}{\partial t}(\mu, t) & =\frac{\partial z}{\partial t}(\mu, t)-\frac{\partial w}{\partial t}(\mu, t) \\
& =\left(A-H_{\Gamma_{G}} C\right) \varphi(\mu, t)
\end{aligned}
$$

The system (1) is $\Gamma_{G}$-detectable, there exists an operator $H_{\Gamma_{G}} \in L\left(\mathcal{O},\left(H^{1 / 2}(\Gamma)\right)^{n}\right)$, such that $\left(A-H_{\Gamma_{G}} C\right)$ generates a strongly continuous semi-group $\left(S_{H_{\Gamma_{G}}}(t)\right)_{t \geq 0}$, which is $\Gamma_{G}$-stable on $\left(H^{1 / 2}(\Gamma)\right)^{n}$, there exists $M_{\Gamma_{G}}, \omega_{\Gamma_{G}}>0$, such that

$$
\|\varphi\|_{\left(H^{1 / 2}(\Gamma)\right)^{n}} \leq\left\|\chi_{\Gamma} \gamma \nabla S_{H_{\Gamma_{G}}}(t)\right\|_{\left(H^{1 / 2}(\Gamma)\right)^{n}}\left\|\varphi_{0}\right\| \leq M_{\Gamma_{G}} e^{-\omega_{\Gamma E G} t}\left\|\varphi_{0}\right\|
$$

With

$$
\varphi_{0}(\mu)=z_{0}(\mu)-w_{0}(\mu),
$$

and hence, we have the following result

$$
\lim _{t \rightarrow \infty}[z(\mu, t)-w(\mu, t)]=0, \mu \in \Gamma .
$$

Definition 5.4: The systems (1)-(2) are $\Gamma_{G}$-observable exponentially, if there exists a dynamical system which is $\Gamma_{G}$-observer exponentially estimate the state gradient of the original system .

Definition 5.5: The system (24) is said to be an identity or full order $\Gamma_{G}$-observer for the systems (1)-(2) [4], if $T_{\Gamma_{G}}=I_{\Gamma_{G}}$ and $Z=W$.

Definition 5.6: The system (24) is said to be a reduced-order $\Gamma_{G}$-observer for the systems (1)-(2), if $Z=\mathcal{O} \oplus W[29]$

Remark 5.7: If the system is a regionally boundary gradient detectable, then it is possible to construct an regionally boundary gradient observer for the original system.

Acknowledgements. Our thanks in advance to the editors and experts for considering this paper to publish in this esteemed journal. The authors appreciate your time and effort in reviewing the manuscript and greatly value your assistant as reviewer for the paper. 


\section{Conclusion}

We have explored the original results devoted to the concept of regional boundary gradient detectability to the state gradient for parabolic distributed system in Hilbert sapce. Then, we have shown that, the possibility to design a dynamic system which is enable to estime the state gradient in sub-region $\Gamma$ of the boundary $\partial \Omega$ bu using detectability and strategic sensors in different situations. Moreover, many problem still opend like the development of these results to case of haperbolic distributed parameter systems as in [25].

\section{References}

[1] Curtain, R. and Zwart, H., "An introduction to infinite dimensional linear system theory". Springer-Verlag, New York, 1995.

[2] Curtain, R. F. and Pritchard, A. J., "Infinite dimensional linear systems theory". Lecture Note in Control and Information Sciences, Springer- Verlag, New York, 1978.

[3] El Jai, A. and Pritchard, A., "Sensors and controls in the analysis of distributed parameter systems". Ellis Harwood Series in Mathematics and Applications, Wiley, New York, 1988.

[4] Al-Saphory, R., Al-Jawari, N. and Al-Janabi, A., "Asymptotic regional gradient full-order observer in distributed parabolic systems". International Journal of Contemporary Mathematical Sciences, Vol. 11, No. 7, 343-358, 2016.

[5] Barje, N., Elarabi Ashhab, M. and Wertz, V., "Observer for linear distributed parameter systems with application to isothermal plug-flow reactor". Intelligent Control and Automatic, Vol. 3, 379-384, 2013.

[6] Hidayat, Z., Babuska, B. and Nunez, A., "Observer for linear distributed parameter systems: a survey". Proceeding of the 2011 IEEE International Symposium on Robotic and Sensors Environments (Rose 2011), Montreal, Canada, 166-171, Sept. 2011.

[7] El Jai, A. and Amouroux, M., "Sensors and observes in distributed parameter systems". International Journal of Control, Vol. 47, No. 1, 333-347, 1988.

[8] El Jai, A., Distributed systems analysis via sensors and actuators. Sensors and Actuators A, Vol. 29, No. 1, 1 $11,1991$.

[9] Al-Saphory, R. Al-Joubory, M. and Jasim, M., "Regional strategic sensors characterizations". Journal of Mathematical and Computational Science. Vol. 3, No. 2, 401-418, 2013.

[10] El Jai, A., Simon, M.C. and Zerrik, E., "Regional observability and sensor structures". Sensors and Actuators A. Vol. 39, No. 2, 95-102, 1993.

[11] El Jai, A., Simon, M. C. Zerrik, E. and Amouroux, M., "Regional observability of a thermal process". IEEE Transaction on Automatic control, Vol. 40, No. 3, 518-521, 1995.

[12] Zerrik, E., Badraoui L. and El Jai, A., "Sensors and regional boundary state reconstruction of parabolic systems". International Journal of Sensors and Actuators, Vol. 75, 102-117, 1999.

[13] Zerrik E., Bourray H., and Boutoulout, A., "Regional boundary observability: a numerical approach". International Journal of Applied Mathematics and Computer Science, Vol. 12, No. 2, 143-151, 2002.

[14] Al-Saphory, R. and El Jai, A., "Sensors structures and regional detectability of parabolic distributed systems". Sensors and Actuators A, Vol. 90, 163-171, 2001. 
[15] Al-Saphory, R. and El Jai, A., "Sensors characterizations for regional boundary detectability in distributed parameter systems". Sensors and Actuators A, Vol. 94, No. 1-2, 1-10, 2001.

[16] Al-Saphory, R., "Strategic sensors and regional exponential observability". ISRN Applied Mathematics. Vol. 2011. Article ID 673052, 1-13, 2011.

[17] Borggaard, J. Burns, J. Surana, A. and Zietsman, L. "Control, estimation and optimization of energy efficient buildings". 2009 American Control Conference. Hyatt regency riverfront, St. Louis, MO, USA, MD, USA. June 10 12,2009 .

[18] Burns, J., Jorggaard, J., Cliff, M. and Zietsman, L, "Optimal sensor design for estimation and optimization of PDE Systems". 2010 American Control Conference. Marriott Waterfront, Baltimore, MD, USA. June 30-July 02, 2010.

[19] Burns, J., "Observers and optimal estimation for PDE systems: sensor location problems", IMA Workshop on Sensor Location in Distributed Parameter Systems, September 6-8, 2017 Minneapolis, MN, USA. 2017.

[20] Al-Saphory, R. Al-Jawari, N. and Al-Janabi, A. "Regional gradient strategic sensors characterizations", Journal of Advances in Mathematics". Vol. 10, No. 6, 3604-3618, 2015.

[21] Al-Saphory, R. Al-Jawari, N. and Al-Qaisi, A. "Regional gradient detectability for infinite dimensional systems". Tikrit Journal of Pure Science. Vol. 15, No. 2, 1-6, 2010.

[22] Al-Saphory, R., Al-Jawari, N., and Al-Janabi, A., "regional boundary gradient strategic sensors Characterizations". Mathematical theory and Modeling, Vol.6, No.1, 2016.

[23] Zerrik, E. and Bourra,y H., "Gradient Observability for diffusion Systems". International Journal of Applied Mathematics and Computer Science. Vol. 13, pp. 139-150, 2003.

[24] Zerrik, E. Bourray ,H., and Badraoui L., "How to reconstruct a gradient for parabolic systems". Conference of MTNS 2000, Perpignan, France, June 19-23, 2000.

[25] Boutoulout, A., Bourray, H., and Khazari, A., "Flux observability for hyperbolic systems". Applied Mathematics and Information Sciences Letters". Vol. 2, No. 1, 13-24, 2014.

[26] Ben Hadid, Rekkab, S., and Zerrik, E., "Sensors and regional gradient observability of hyperbolic systems". Intelligent Control and Automation 3, 78-89, 2012.

[27] Zerrik, E. and Ouzahra M., "Output stabilization for infinite-dimensional bilinear systems", International Journal of Applied Mathematics and computer Science, Vol. 15, No. 2, 187-195, 2005.

[28] Brezis H., "Analyse Fonctionnalle". Theorie et applications. 2em tirage. Masson, Paris, France,1987.

[29] Al-Saphory, R. and Al-Mullah, Sh., "Regional exponential reduced observability in distributed parameter systems". Journal of Advances in Mathematics.Vol. 11, No. 4,5050-5068, 2015. 